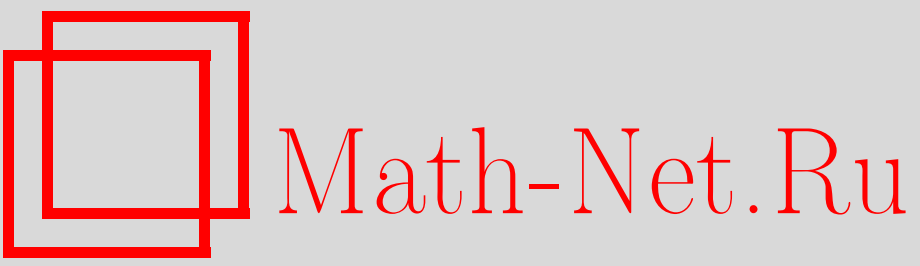

А. В. Жибер, В. В. Соколов, Новый пример гиперболического нелинейного уравнения, обладающего интегралами, ТМФ, 1999, том 120, номер 1, 20-26

DOI: https://doi.org/10.4213/tmf757

Использование Общероссийского математического портала Math-Net.Ru подразумевает, что вы прочитали и согласны с пользовательским соглашением

http://www.mathnet.ru/rus/agreement

Параметры загрузки:

IP: 52.205 .19 .152

26 апреля 2023 г., 16:49:26 
ТЕОРЕТИЧЕСКАЯ

И МАТЕМАТИЧЕСКАЯ

ФИЗИКА

Том 120, № 1

июль, 1999

(C) $\mathbf{1 9 9 9}$ г.

А.В. Жибер*, В.В. Соколов ${ }^{\dagger}$

\section{НОВЫЙ ПРИМЕР ГИПЕРБОЛИЧЕСКОГО НЕЛИНЕЙНОГО УРАВНЕНИЯ, ОБЛАДАЮШЕГО ИНТЕГРАЛАМИ}

Найден новый важный пример в классической задаче классификации нелинейных гиперболических уравнений, обладающих интегралами. Кроме того, в случае общего положения получена формула, описывающая расщепление правой части таких уравнений по первым производным.

\section{ВВЕДЕНИЕ}

Стандартным примером точно интегрируемого нелинейного уравнения в частных производных является уравнение Лиувилля $u_{x y}=\exp u$. Изучение формальных свойств этого уравнения привело [1-3] к двум близким определениям интегрируемости для уравнений

$$
u_{x y}=F\left(x, y, u, u_{x}, u_{y}\right)
$$

Согласно определению 1 уравнение типа уравнения Лиувилля характеризуется существованием параметрически заданного решения вида

$$
\begin{aligned}
& x=A\left(\alpha, \beta, p, p^{\prime}, \ldots, q, q^{\prime}, \ldots\right), \\
& y=B\left(\alpha, \beta, p, p^{\prime}, \ldots, q, q^{\prime}, \ldots\right), \\
& u=C\left(\alpha, \beta, p, p^{\prime}, \ldots, q, q^{\prime}, \ldots\right),
\end{aligned}
$$

зависящего от произвольных функций $p(\alpha), q(\beta)$ и конечного числа их производных.

Определение 2 требует существования у (1) нетривиальных $x$ - и $y$-интегралов. Задача полной классификации уравнений, обладаюших интегралами, в настояший момент доведена до конца только в случае, если порядки интегралов не превышают двух

\footnotetext{
${ }^{*}$ Институт механики Уфимского научного центра РАН, Уфа, Россия

${ }^{\dagger}$ Институт теоретической физики им. Л.Д. Ландау РАН, Черноголовка, Россия. Е-mail: sokolov@landau.ac.ru
} 
(см. $[4,5])$. Полученный список совпадает со списком уравнений, содержащимся в [2] (см. также [6]), и состоит (с точностью до точечных замен) из следующих уравнений:

$$
\begin{gathered}
u_{x y}=e^{u}, \\
u_{x y}=u_{x} e^{u}, \\
u_{x y}=e^{u} \sqrt{\left(u_{x}^{2}-4\right)}, \\
u_{x y}=\left(\frac{1}{(u-x)}+\frac{1}{(u-y)}\right) u_{x} u_{y}, \\
u_{x y}=-\frac{2}{(x+y)} \sqrt{u_{x}} \sqrt{u_{y}}, \\
u_{x y}=\psi(u) \beta\left(u_{x}\right) \bar{\beta}\left(u_{y}\right), \quad(\ln \psi)^{\prime \prime}=\psi^{2}, \quad \beta \beta^{\prime}=-u_{x}, \quad \bar{\beta} \bar{\beta}^{\prime}=-u_{y}, \\
u_{x y}=\frac{1}{u} \beta\left(u_{x}\right) \bar{\beta}\left(u_{y}\right), \quad \beta \beta^{\prime}+c \beta=-u_{x}, \quad \bar{\beta} \bar{\beta}^{\prime}+c \bar{\beta}=-u_{y}, \\
u_{x y}=\frac{1}{(x+y)} \beta\left(u_{x}\right) \bar{\beta}\left(u_{y}\right), \quad \beta \beta^{\prime}+\beta+1=0, \quad \bar{\beta} \bar{\beta}^{\prime}+\bar{\beta}+1=0 .
\end{gathered}
$$

Конечно, все дифференциальные уравнения для $\psi(u), \beta\left(u_{x}\right)$ и $\bar{\beta}\left(u_{y}\right)$ легко интегрируются. Однако, если приводить явные формулы, то размер списка несколько увеличивается из-за необходимости рассматривать частные решения по отдельности. В частности, в нем неявно содержится уравнение

$$
u_{x y}=\frac{1}{u} \sqrt{1-u_{x}^{2}} \sqrt{1-u_{y}^{2}},
$$

которое является вырождением интегрируемого методом обратной задачи рассеяния уравнения

$$
u_{x y}=\operatorname{sn}(u) \sqrt{1-u_{x}^{2}} \sqrt{1-u_{y}^{2}},
$$

где $\operatorname{sn}(u)$ - эллиптический синус Якоби. Последнее уравнение в значительно более громоздкой форме впервые возникло в работе [7].

Все уравнения приведенного выше списка удовлетворяют как определению 1, так и определению 2. Долгое время казалось, что:

1) уравнений, обладающих интегралами порядка, более высокого чем два, не существует;

2) определения 1 и 2 эквивалентны.

В настоящей работе приводится пример уравнения (см. формулу (20) ниже), обладающего интегралами третьего порядка. Можно доказать, что это уравнение не удовлетворяет определению 1.

Нам удалось найти это уравнение, используя некоторый специальный анзац для правой части уравнения (1). Сам анзац возникает при исследовании случая "общего положения" для уравнений, обладающих интегралами. А именно, мы доказываем, что уравнение (с точностью до точечной замены) должно иметь следующий вид:

$$
u_{x y}=\frac{R_{1} A \bar{A}+R_{2} A+R_{3} \bar{A}+R_{4}}{A_{u_{x}} \bar{A}_{u_{y}}},
$$


где $R_{i}$ - некоторые функции, зависяшие от $x, y, u ; A$ зависит только от $x$ и $u_{x}$, а $\bar{A}$ - от $y$ и $u_{y}$. Для всех уравнений приведенного выше списка $R_{2}=R_{3}=R_{4}=0$. Для уравнения (20) (см. ниже) это не так.

\section{1. ВЫВОД ФОРМУЛ ДЛЯ ЧАСТНЫХ ПРОИЗВОДНЫХ ОТ ПРАВОЙ ЧАСТИ УРАВНЕНИЯ}

Будем использовать обозначения

$$
\begin{aligned}
& u_{1}=u_{x}, \quad u_{2}=u_{x x}, \quad u_{3}=u_{x x x}, \ldots, \\
& \bar{u}_{1}=u_{y}, \quad \bar{u}_{2}=u_{y y}, \quad \bar{u}_{3}=u_{y y y}, \ldots .
\end{aligned}
$$

Операторы $D$ и $\bar{D}$ полного дифференцирования по $x$ и по $y$ в силу уравнения (1) имеют вид

$$
D=\frac{\partial}{\partial x}+\sum_{i=0}^{\infty} u_{i+1} \frac{\partial}{\partial u_{i}}+\sum_{i=1}^{\infty} \bar{D}^{i-1}(F) \frac{\partial}{\partial \bar{u}_{i}}
$$

и

$$
\bar{D}=\frac{\partial}{\partial y}+\sum_{i=0}^{\infty} \bar{u}_{i+1} \frac{\partial}{\partial \bar{u}_{i}}+\sum_{i=1}^{\infty} D^{i-1}(F) \frac{\partial}{\partial u_{i}} .
$$

Функция $W\left(x, y, u, u_{1}, \ldots, u_{p}\right)$ называется $x$-интегралом, если $\bar{D}(W)=0$. Последнее соотношение означает, что, подставив в $W$ любое решение уравнения (1), мы получим некоторую (вообще говоря, разную для разных решений) функцию, зависяшую только от $x$.

Аналогично $y$-интеграл - это функция $\bar{W}\left(x, y, u, \bar{u}_{1}, \ldots, \bar{u}_{\bar{p}}\right)$, удовлетворяюшая соотношению $D(\bar{W})=0$.

В работе [4] (см. также [8]) доказано, что если (1) обладает интегралами, то

$$
\begin{aligned}
& \frac{\partial F}{\partial u_{1}}=\bar{D} K\left(x, y, u, u_{1}, \ldots, u_{p}\right), \\
& \frac{\partial F}{\partial \bar{u}_{1}}=D \bar{K}\left(x, y, u, \bar{u}_{1}, \ldots, \bar{u}_{\bar{p}}\right)
\end{aligned}
$$

для некоторых функций $K$ и $\bar{K}$. Минимальное из чисел $p$, для которых выполняется (6), является важной характеристикой уравнения (1). Если это число равно $r$, то мы будем говорить, что уравнение имеет тип $r$ по переменным $u_{i}$. Аналогично определяется тип уравнения $\bar{r}$ по переменным $\bar{u}_{i}$.

Кроме условий (6), (7), имеется еще одно очень полезное симметричное соотношение

$$
\frac{\partial F}{\partial u_{1}} \frac{\partial F}{\partial \bar{u}_{1}}+\frac{\partial F}{\partial u}=\bar{D} L\left(x, y, u, u_{1}, \ldots, u_{s}\right)=D \bar{L}\left(x, y, u, \bar{u}_{1}, \ldots, \bar{u}_{\bar{s}}\right) .
$$

Предположим, что $r>2, \bar{r}>2$. В этом случае (см. [4]) вид функций $K$ и $\bar{K}$ можно уточнить:

$$
\begin{aligned}
& \frac{\partial F}{\partial u_{1}}=\bar{D}\left(\ln \left(u_{r}+g\left(x, y, u, u_{1}, \ldots, u_{r-1}\right)\right)\right), \\
& \frac{\partial F}{\partial \bar{u}_{1}}=D\left(\ln \left(\bar{u}_{\bar{r}}+\bar{g}\left(x, y, u, \bar{u}_{1}, \ldots, \bar{u}_{\bar{r}-1}\right)\right)\right) .
\end{aligned}
$$


Дифференцируя равенства (9) по переменной $u_{r-1}$, можно показать [4], что

$$
\frac{\partial F}{\partial u_{1}} \frac{\partial F}{\partial \bar{u}_{1}}+\frac{\partial F}{\partial u}=(1-r) D\left(\frac{\partial F}{\partial u_{1}}\right)+\bar{D}\left(\alpha\left(x, y, u, u_{1}\right) u_{2}+\beta\left(x, y, u, u_{1}\right)\right)
$$

для некоторых функций $\alpha, \beta$. Аналогично имеем

$$
\frac{\partial F}{\partial u_{1}} \frac{\partial F}{\partial \bar{u}_{1}}+\frac{\partial F}{\partial u}=(1-\bar{r}) \bar{D}\left(\frac{\partial F}{\partial \bar{u}_{1}}\right)+D\left(\bar{\alpha}\left(x, y, u, \bar{u}_{1}\right) \bar{u}_{2}+\bar{\beta}\left(x, y, u, \bar{u}_{1}\right)\right)
$$

Приравнивая в (10) коэффициенты при $u_{2}$, получаем

$$
(r-1) \frac{\partial^{2} F}{\partial u_{1}^{2}}=\bar{D}(\alpha)+\frac{\partial F}{\partial u_{1}} \alpha
$$

Это равенство можно проинтегрировать по $u_{1}$ (поскольку правая часть имеет вид $\partial \bar{D}(S) / \partial u_{1}$, где $\left.\alpha=\partial S / \partial u_{1}\right)$. В результате получится соотношение вида

$$
\frac{\partial F}{\partial u_{1}}+\bar{D} \varphi\left(x, y, u, u_{1}\right)=\bar{f}\left(x, y, u, \bar{u}_{1}\right)
$$

Аналогично из (11) извлекается соотношение

$$
\frac{\partial F}{\partial \bar{u}_{1}}+D \bar{\varphi}\left(x, y, u, \bar{u}_{1}\right)=f\left(x, y, u, u_{1}\right)
$$

Формулы (12), (13) инвариантны относительно точечных замен

$$
x=P(X), \quad y=Q(Y), \quad u=R(X, Y, U),
$$

сохраняющих класс уравнений (1). А именно, правая часть $G\left(X, Y, U, U_{X}, U_{Y}\right)$ преобразованного уравнения удовлетворяет соотношениям

$$
\frac{\partial G}{\partial U_{1}}+\bar{D}\left(\varphi+\ln \left(R_{U}\right)\right)=Q^{\prime} \bar{f}, \quad \frac{\partial G}{\partial \bar{U}_{1}}+D\left(\bar{\varphi}+\ln \left(R_{U}\right)\right)=P^{\prime} f .
$$

Поскольку функции $\varphi$ и $\bar{\varphi}$ определены с точностью до слагаемых, зависяших от $x, y, u$, можно считать, что они являются абсолютными инвариантами относительно замен (14).

По-видимому, наличие пары соотношений $(12),(13)$ является общим фактом в теории уравнений, обладаюших интегралами. Мы получили (12) при $r>2$. В случае, когда $r<2$, само условие (6) имеет вид (12) с $\bar{f}=0$. Таким образом, только случай $r=2$ требует специального рассмотрения. Хотя здесь мы пока не умеем доказывать, что соотношение вида (12) сушествует всегда, во многих частных случаях оно возникает самым неожиданным образом.

Ниже мы, пользуясь условиями совместности для соотношений (12), (13), уточняем возможньй вид правой части уравнения (1). 


\section{2. ФОРМУЛА ДЛЯ ПРАВОЙ ЧАСТИ УРАВНЕНИЯ (1)}

Дифференцируя $(12)$ по $\bar{u}_{1},(13)$ по $u_{1}$ и вычитая одно полученное выражение из другого, находим, что

$$
\bar{f}_{\bar{u}_{1}}+\bar{\varphi}_{u}+\bar{\varphi}_{\bar{u}_{1}}\left(\bar{f}-\varphi_{y}-\bar{u}_{1} \varphi_{u}\right)=f_{u_{1}}+\varphi_{u}+\varphi_{u_{1}}\left(f-\bar{\varphi}_{x}-u_{1} \bar{\varphi}_{u}\right) .
$$

Применяя к (15) оператор $\Delta=\partial^{2} / \partial u_{1} \partial \bar{u}_{1}$ и обозначая $\psi=\varphi_{u_{1}}, \bar{\psi}=\bar{\varphi}_{\bar{u}_{1}}$, получаем

$$
\psi_{u_{1}} \bar{\psi}_{x}+\bar{\psi}_{u} \frac{\partial}{\partial u_{1}}\left(u_{1} \psi\right)=\bar{\psi}_{\bar{u}_{1}} \psi_{y}+\psi_{u} \frac{\partial}{\partial \bar{u}_{1}}\left(\bar{u}_{1} \bar{\psi}\right)
$$

Если $\psi_{u_{1}} \bar{\psi}_{\bar{u}_{1}} \neq 0$, то поделим равенство (16) на это произведение и снова применим оператор $\Delta$. В результате имеем

$$
\frac{\partial}{\partial \bar{u}_{1}}\left(\frac{\bar{\psi}_{u}}{\bar{\psi}_{\bar{u}_{1}}}\right) \frac{\partial}{\partial u_{1}}\left(\frac{\frac{\partial}{\partial u_{1}}\left(u_{1} \psi\right)}{\psi_{u_{1}}}\right)=\frac{\partial}{\partial u_{1}}\left(\frac{\psi_{u}}{\psi_{u_{1}}}\right) \frac{\partial}{\partial \bar{u}_{1}}\left(\frac{\frac{\partial}{\partial \bar{u}_{1}}\left(\bar{u}_{1} \bar{\psi}\right)}{\bar{\psi}_{\bar{u}_{1}}}\right) .
$$

Рассмотрим самый невырожденный случай, когда все сомножители в (17) не равны нулю. Тогда в этом выражении можно разделить переменные, что дает пару уравнений вида

$$
\psi_{u}=\alpha\left(\psi+u_{1} \psi_{u_{1}}\right)+\gamma \psi_{u_{1}}, \quad \bar{\psi}_{u}=\alpha\left(\bar{\psi}+\bar{u}_{1} \bar{\psi}_{\bar{u}_{1}}\right)+\epsilon \bar{\psi}_{\bar{u}_{1}},
$$

где $\alpha, \gamma, \epsilon$-некоторые функции от $x, y, u$.

Подставляя полученные выражения для $\psi_{u}$ и $\bar{\psi}_{u}$ в (16) и расшепляя переменные, получаем

$$
\psi_{y}=\epsilon\left(\psi+u_{1} \psi_{u_{1}}\right)+\mu \psi_{u_{1}}, \quad \bar{\psi}_{x}=\gamma\left(\bar{\psi}+\bar{u}_{1} \bar{\psi}_{\bar{u}_{1}}\right)+\mu \bar{\psi}_{\bar{u}_{1}} .
$$

Совместность уравнений (18), (19) эквивалентна соотношениям

$$
\epsilon_{u}=\alpha_{y}, \quad \gamma_{u}=\alpha_{x}, \quad \gamma_{y}=\epsilon_{x}, \quad \mu_{u}-\gamma_{y}=\gamma \epsilon-\alpha \mu .
$$

Учитывая эти равенства, можно проверить, что общее решение системы (18), (19) задается формулой

$$
\psi=H_{u} \Phi(x, D(H)), \quad \bar{\psi}=H_{u} \bar{\Phi}(y, \bar{D}(H)),
$$

где $\Phi, \bar{\Phi}_{\text {и }} H(x, y, u)$ - произвольные функции.

После точечной замены $H(x, y, u) \rightarrow u$ имеем $\psi=\psi\left(x, u_{1}\right), \bar{\psi}=\bar{\psi}\left(y, \bar{u}_{1}\right)$. Так как $\varphi$, $\bar{\varphi}$ определены с точностью до сдвига на функции от $x, y, u$, можно считать, что $\varphi=$ $\varphi\left(x, u_{1}\right), \bar{\varphi}=\bar{\varphi}\left(y, \bar{u}_{1}\right)$. Теперь соотношение (15) принимает вид

$$
\bar{f}_{\bar{u}_{1}}+\bar{\varphi}_{\bar{u}_{1}} \bar{f}=f_{u_{1}}+\varphi_{u_{1}} f .
$$

Разделяя переменные, находим

$$
\bar{f}=\frac{R_{1} A+R_{2}}{A_{u_{1}}}, \quad f=\frac{R_{1} \bar{A}+R_{3}}{\bar{A}_{\bar{u}_{1}}},
$$

где $A_{u_{1}}=\exp \varphi, \bar{A}_{\bar{u}_{1}}=\exp \bar{\varphi}$. Наконец, соотношения (12), (13) дают формулу (3).

Для полного исследования совместности системы (12), (13) нужно рассмотреть еще случаи, когда один или несколько множителей, на которые мы делили при расщеплении соотношения (15), равен нулю. В принципе это нетрудно сделать, но формулы оказываются довольно громоздкими, и мы их здесь не приводим. 


\section{3. ПРИМЕР}

Если предположить в дополнение к (3), что тип уравнения определяется равенствами $r=\bar{r}=2$, а числа $s, \bar{s}$, фигурирующие в условии (8), равны единице, то прямые вычисления приводят к весьма любопытному уравнению

$$
u_{x y}=\frac{f^{2}(f-1) \bar{f}(\bar{f}-1)^{2}}{(6 u+y)}+\frac{\bar{f}^{2}(\bar{f}-1) f(f-1)^{2}}{(6 u+x)}
$$

где функции $f\left(u_{x}\right)$ и $\bar{f}\left(u_{y}\right)$ определяются как решения кубических уравнений

$$
\frac{1}{3} f^{3}-\frac{1}{2} f^{2}=u_{x}, \quad \frac{1}{3} \bar{f}^{3}-\frac{1}{2} \bar{f}^{2}=u_{y} .
$$

Легко видеть, что уравнение (20) имеет вид (3), где

$$
\begin{aligned}
A & =f, \quad \bar{A}=\bar{f}, \quad R_{1}=\frac{1}{(6 u+y)}+\frac{1}{(6 u+x)}, \\
R_{2} & =-\frac{1}{(6 u+y)}, \quad R_{3}=-\frac{1}{(6 u+x)}, \quad R_{4}=0 .
\end{aligned}
$$

Уравнение (20) обладает интегралами третьего порядка: $x$-интеграл задается формулой

$$
\begin{aligned}
W= & D\left(\ln \left(u_{2}-\frac{f^{4}(f-1)^{2}}{6 u+y}-\frac{f^{2}(f-1)^{4}}{6 u+x}\right)-\ln (f)-\ln (f-1)\right)- \\
& -\frac{f^{2}(f-1)}{6 u+y}-\frac{f(f-1)^{2}}{6 u+x},
\end{aligned}
$$

а $y$-интеграл получается из него заменой $u_{2} \rightarrow \bar{u}_{2}, f \rightarrow \bar{f}, D \rightarrow \bar{D}$.

Можно проверить, что функция

$$
u=-\frac{x+y}{12}+\frac{A}{12 \sqrt{B}}
$$

где

$$
\begin{aligned}
A= & 2(X+Y)^{3}+3(x-y)(X+Y)(\bar{P}-P)+(x-y)^{2}(\bar{Q}+Q), \\
B= & (x-y)^{2}(\bar{Q}+Q)^{2}-2(x-y)(\bar{P}-P)\left(2(\bar{P}-P)^{2}-\right. \\
& -3(\bar{Q}+Q)(X+Y))+4(X+Y)^{3}(\bar{Q}+Q)-3(X+Y)^{2}(\bar{P}-P),
\end{aligned}
$$

удовлетворяет уравнению (20). Здесь $X(x)$ и $Y(y)$ - произвольные функции, а функции $P(x), Q(x), \bar{P}(y)$ и $\bar{Q}(y)$ связаны с ними дифференциальными соотношениями

$$
X^{\prime}=P^{\prime 2}, \quad X^{\prime}=Q^{\prime 3}, \quad Y^{\prime}=\bar{P}^{\prime 2}, \quad Y^{\prime}=\bar{Q}^{\prime 3} .
$$


Если бы эти дифференциальные связи можно было разрешить локальной подстановкой вида

$$
\begin{aligned}
X & =A\left(\alpha, \beta, p, p^{\prime}, \ldots, q, q^{\prime}, \ldots\right), \\
P & =B\left(\alpha, \beta, p, p^{\prime}, \ldots, q, q^{\prime}, \ldots\right), \\
Q & =C\left(\alpha, \beta, p, p^{\prime}, \ldots, q, q^{\prime}, \ldots\right), \\
Y & =\bar{A}\left(\alpha, \beta, p, p^{\prime}, \ldots, q, q^{\prime}, \ldots\right), \\
\bar{P} & =\bar{B}\left(\alpha, \beta, p, p^{\prime}, \ldots, q, q^{\prime}, \ldots\right), \\
\bar{Q} & =\bar{C}\left(\alpha, \beta, p, p^{\prime}, \ldots, q, q^{\prime}, \ldots\right),
\end{aligned}
$$

то мы получили бы из (21) формулу вида (2). Однако легко видеть, что локальным образом этим связям удовлетворить невозможно.

Благодарности. Мы благодарны С. П. Цареву и А. Б. Шабату за полезные обсуждения. Исследования были выполнены при финансовой поддержке Российского фонда фундаментальных исследований (грант 99-01-00294).

\section{Список литературы}

[1] G. Darboux. Leçons sur la theorie general des surface. Tome II. Paris: Hermann, 1915.

[2] E. Goursat. Leçons sur l'intégration des équations aux dérivées partielles du second ordre à deux variables indépendantes. Paris: Hermann. Tome I, 1896; Tome II, 1898.

[3] E. Vessiot. J. Math. Pure Appl. 1939. V. 18. P. 1-61.

[4] А. В. Ж ибер. Изв. РАН. Сер. матем. 1994. Т. 58. № 4. С. 33-54.

[5] А. В. Жибер. Высшие симметрии уравнений типа Лиувилля. В сб.: Труды Всероссийской научной конф. "Физика конденсированного состояния". Т. 1. Математические методы физики. Ред. К.Б. Сабитов (отв. за выпуск), Ф.Х. Мукминов, В.Ф. Волкодавов, С. В. Хабиров. 1997. Стерлитамак: Стерлитамакский филиал АН РБ, СГПИ, 1997. С. 54-57.

[6] М. Ю. Звягин. ДАН СССР. 1991. Т. 316. № 1. С. 36-40.

[7] А. Б. Борисов, С. А. Зыков. ТМФ. 1998. Т. 115. № 2. С. 199-214.

[8] А. В. Жибер, А. Б. Шабат. ДАН СССР. 1984. Т. 277. № 1. С. 29-33.

Поступила в редакцию 30.Х.1998 г., после доработки 19.XI.1998 г. 